\title{
Short-term impact of the smokefree legislation in England on emergency hospital admissions for asthma among adults: a population-based study
}

\author{
Michelle Sims, ${ }^{1}$ Roy Maxwell, ${ }^{2}$ Anna Gilmore ${ }^{1}$
}

- Additional material is published online only. To view please visit the journal online (http://dx.doi.org/10.1136/ thoraxjnl-2012-202841).

${ }^{1}$ School for Health, and the UK Centre for Tobacco Control Studies, University of Bath, Bath, UK

${ }^{2}$ South West Public Health Observatory, Bristol, UK

\section{Correspondence to} Dr Michelle Sims, Tobacco Control Research Group, School for Health, University of Bath, Claverton Down, Bath BA2 7AY, UK;

m.sims@bath.ac.uk

Received 9 October 2012 Revised 20 February 2013 Accepted 26 February 2013 Published Online First 16 April 2013
To cite: Sims M,

Maxwell R, Gilmore A.

Thorax 2013;68:619-624.

\begin{abstract}
Background Comprehensive smokefree laws have now been introduced in several jurisdictions. Few studies have examined the association between smokefree laws and asthma in adults and these have limitations, such as lacking appropriate adjustment for long-term trends or having limited statistical power due to small study populations. This study addresses these limitations and evaluates the short-term impact of smokefree legislation in England. It aims to investigate whether the introduction of smokefree legislation on 1 July 2007 was associated with an immediate reduction in emergency hospital admissions for asthma in the adult population, and whether any association differs across regions.
\end{abstract}

Methods We identified monthly numbers of emergency admissions for asthma (primary diagnosis, 10th revision of the International Classification of Diseases code $J 45$ and J46) in the nine Government Office Regions from April 1997 to December 2010 in the population aged 16 and over. A generalised additive model was fitted that adjusted for seasonality, variation in population size and region-specific, non-linear, long-term trends.

Results Smokefree legislation was associated with an immediate $4.9 \%$ (95\% Cl $0.6 \%$ to $9.0 \%$ ) reduction in emergency admissions for asthma in the adult population. This implies that approximately 1900 emergency admissions for asthma were prevented in each of the first 3 years after legislation was introduced. The reduction in admissions did not vary significantly across regions.

Conclusions Our findings add to the expanding body of evidence that smokefree policies are associated with positive health outcomes. Further research evaluating the impact of legislation in other jurisdictions is needed to support these findings.

\section{INTRODUCTION}

Second hand smoke (SHS) exposure has significant adverse health effects on the adult respiratory system, ${ }^{1}$ with current evidence suggesting that it contributes to the onset ${ }^{2}$ and exacerbation of asthma ${ }^{3}{ }^{4}$ and to poorer asthma health outcomes. ${ }^{5}$ Exposure also appears to be associated with a higher risk of hospital admissions for asthma within a month after exposure. ${ }^{6}$ These findings are supported by studies that show the deleterious effects of SHS exposure on airflow and airway responsiveness in those with asthma. ${ }^{3-9}$

Comprehensive smokefree laws have now been introduced in several jurisdictions. Evidence that such legislation reduces SHS exposure (measured
Key messages

What is the key question?

- Was the introduction of smokefree legislation in England associated with an immediate reduction in emergency hospital admissions for asthma in the adult population?

What is the bottom line?

- In this population-based study, emergency admissions for asthma in adults decreased following the introduction of smokefree legislation in England.

\section{Why read on?}

- This study, the largest of its kind and addressing limitations of previous studies, describes the positive effect that a public health intervention can have on hospital admissions for asthma.

using specific biomarkers) among adults in hospitality workers and patrons ${ }^{10}$ and the general population of non-smokers ${ }^{11-13}$ is accumulating. There is also a growing body of evidence documenting the immediate health benefits of smokefree laws, focusing primarily on hospital admissions for heart attacks. ${ }^{14}$ The association between legislation and asthma admissions among children, ${ }^{15-17}$ adults ${ }^{17-19}$ and the overall population (children and adults combined) ${ }^{20} 21$ has not been as thoroughly investigated. While the results from existing studies have recently been combined in a meta-analysis, which concluded that comprehensive smokefree laws are associated with a $24 \%(95 \%$ CI $13 \%$ to $34 \%)$ decrease in asthma admissions, ${ }^{14}$ this estimate includes studies based on widely differing age groups. Only a few studies have examined impacts in adults and collectively the findings from these studies are inconclusive. For example, studies in Ireland ${ }^{19}$ Kentucky ${ }^{17}$ and Delaware $^{22}$ reported statistically significant reductions in hospital asthma admissions after the introduction of smoke-free legislation of $40 \%, 24 \%$ and $5 \%$ respectively, while a New Zealand ${ }^{18}$ study reported a non-statistically significant decline of $16 \%$. Moreover some of these studies have limited statistical power due to small study populations and it is unclear whether underlying long-term trends in asthma admissions were properly controlled for. 
Two of these studies, in New Zealand ${ }^{18}$ and Kentucky, ${ }^{17}$ used a Poisson regression model and adjusted for long-term trends by including a linear term for time, but there is no mention of whether the linearity assumption in the long-term trend was tested. A study in Ireland ${ }^{19}$ applied a Poisson regression model to admissions data collected for 2 years pre and post legislation, which was adjusted for differences in potential confounding factors (eg, flu and temperature) between the two time periods, but did not adjust for long-term trends which would have captured temporal changes in other risk factors. The study in Delaware also applied a Poisson regression model which adjusted for seasonal effects and population size, but the estimated effect of 5\% did not include adjustment for a long-term trend. ${ }^{22}$ Incorrect assumptions about the shape of the trend or ignoring it completely can result in a biased estimate of the effect of a ban. ${ }^{23}$

We addressed the limitations of earlier studies by investigating the short-term impact of legislation in England, where virtually all enclosed public places and workplaces became smoke free on 1 July 2007. The prevalence of asthma in England is among the highest in the world, with approximately $5.9 \%$ of the population having asthma. ${ }^{24}$ Furthermore, there are stark regional differences in emergency hospital admissions for asthma across England that have widened in recent years. ${ }^{25}$ Reducing hospital admissions for asthma is therefore an objective of the current UK government's strategy for people with asthma in England. The aims of this study were to investigate whether the introduction of smokefree legislation was associated with an immediate reduction in emergency hospital admissions for asthma in the adult population, and whether any association differs across regions. We do not consider children for two reasons. First, their exposure predominantly occurs in non-public places, such as their home, and existing evidence indicates that they were less directly affected by the legislation $^{26} 27$ compared with non-smoking adults in whom legislation has been linked to reductions in SHS exposure. ${ }^{13}$ Second, recently published work has already examined impacts in children. ${ }^{28}$

\section{METHODS}

\section{Hospital admission data}

Hospital Episode Statistics (HES) data provide routinely collected information on all patients who receive care provided by the National Health Service (NHS) in England. ${ }^{29}$ Each completed record in HES, a 'finished consultant episode' (FCE), is a continuous period of time a patient spends while under the care of one consultant within one healthcare provider. We identified hospital emergency admissions for adult asthma, selecting all emergency admission FCEs in those aged 16 years and over, resident in England, with an admission date between 1 April 1997 and 31 December 2010 and a primary diagnosis of asthma (10th revision of the International Classification of Diseases (ICD-10) code J45 or $\mathrm{J} 6^{30}$ ). ICD-10 coding was introduced by the NHS in England in 1995. To allow for any irregularities in coding that followed the change from ICD-9 to ICD-10, the start date of the study was delayed until 1997. The end date of 31 December 2010 was the latest date for which data were available and no longer considered provisional. Our study period therefore included 3 years and 6 months post-intervention and 10 years and 3 months pre-intervention data. Only the first FCE (known as the finished consultant admission episode) of a patient's spell in hospital was used, and these were aggregated into monthly numbers of emergency admissions according to the Government Office Region of residence of the patient (East Midlands, East of England, London, North East England, North West England, South East England, South West England, West Midlands, and Yorkshire and the Humber). Thus our data comprised
165 months for each of the nine regions (1485 observations in total). Although smoking status can be recorded in HES using ICD codes, this information is not routinely recorded and consequently we cannot accurately differentiate between smokers and non-smokers. Records with missing age values were excluded from the analysis (less than $0.05 \%$ of records).

\section{Statistical analysis}

We tested the hypothesis that there was an immediate change in the number of emergency admissions for asthma following the introduction of smokefree legislation using a Poisson generalised additive model of the monthly admissions.

To model non-linear seasonal fluctuations in admissions due to weather conditions and influenza we included penalised cubic regression splines for both. A penalised cyclic cubic spline for month was also included to capture any additional fluctuations due to seasonally varying factors other than temperature and influenza. Monthly mean temperatures for a government office region were derived by obtaining monthly mean temperature records for meteorological office stations within the region and taking the average. We obtained monthly rates of hospital admissions due to influenza for each government office region from the HES database, restricting data to admission episodes of patients aged 16 and over with a primary diagnosis of influenza (ICD-10 code J10 or J11).

Variation in monthly rates of emergency admissions for asthma and non-linearity in long-term trends among the nine government office regions was modelled using a categorical variable for region and an interaction between the categorical variable for region and a penalised cubic regression spline for time (the time series of monthly counts).

The introduction of smokefree legislation was included in the model as a binary predictor variable, with 1 assigned to admissions from July 2007 onwards and 0 assigned to admissions before July 2007. We therefore investigated whether or not there was an immediate change in emergency admissions for asthma after the smokefree legislation was introduced. We also examined whether or not the magnitude of the immediate impact of legislation varied by region by adding an interaction term between the binary smokefree legislation predictor and the categorical variable for region.

To account for the variation in the number of days per month and changes in the population size over time, number of days and population size were included as offset variables; that is, the natural logarithms of these variables were included as predictors with their regression coefficients fixed at 1 . Mid-year estimates and projections of the population aged 16 and over in each government office region were obtained from the UK's Office of National Statistics. Monthly population figures were obtained by linearly interpolating mid-year population estimates for each government office region for 1996 to 2010 and population projections for 2011. Overdispersion was detected and SEs were corrected using a quasi-Poisson model.

An exploration of this model showed some short-term autocorrelation in the residuals within each region. In the final model, we therefore included a residual autoregressive structure of order $1(\mathrm{AR}(1))$ for each region to capture this short-term serial correlation. We generated autocorrelation and partial autocorrelation plots to compare this model with one without the autocorrelation structure for the residuals to confirm that the $\mathrm{AR}(1)$ structure was appropriate.

To correct for differences in age and gender distribution across regions and for changes in these distributions over time, we replaced monthly counts as the outcome variable with 
monthly age-sex standardised counts, using the 2001 census population as the reference; we then refitted the model but did not include population size as an offset term. The difference in the estimated immediate impact of smokefree legislation between the model using population-adjusted counts or age-sex standardised counts was negligible. Consequently the results are not shown for this model.

The generalised additive model based on the time series of monthly admissions was used to predict the number of emergency admissions for asthma that were prevented as a result of smokefree legislation in the first, second and third year of implementation following an approach described elsewhere. ${ }^{31}$

Models were fitted in R.13.0 using the gam and gamm functions from the library mgcv. ${ }^{32}$ All tests were two sided and performed at the $5 \%$ level of statistical significance.

\section{Sensitivity analyses}

To provide further support for our findings we performed two sensitivity analyses. For the first analysis we randomly selected 20 false smokefree legislation dates between January 2005 and January 2008. For each false date, we fitted the model but replaced the binary smokefree predictor with a binary predictor coded as 0 for admissions before this date, and 1 for admissions from this date onwards. In the second analysis we investigated the sensitivity of our results to variations in the length of the pre-legislation and post-legislation period. We fitted the model to data with a postlegislation period of 1, 2 or 3 years and a pre-legislation period of 6-10 years. These ranges were selected based on data availability and what was allowed by the model fitting. If our model specification is appropriate we would not expect significant changes at the false dates and the estimated association between smokefree legislation and admissions for asthma would be robust to variations in the length of the pre-legislation and post-legislation periods.

\section{RESULTS}

Impact of smokefree legislation

Over the study period 1997 to 2010, 502000 emergency admissions among adults aged 16 and over had a primary diagnosis of asthma. Although seasonal patterns were similar across regions with higher admission rates in mid winter than in summer, there were notable differences in the level of, and long-term trends in, admission rates for asthma across regions (figure 1). For example, average monthly admission rates for the period under study ranged from 0.059 per 1000 adults in the South East to 0.089 per 1000 adults in Yorkshire and the Humber.

After adjusting for seasonality, variation in population size and long-term trends, the introduction of smokefree legislation in July 2007 was associated with a statistically significant $4.9 \%$ drop ( 1 minus the exponential of -0.05 ; table 1$)(95 \% \mathrm{CI}$ 0.6 to 9.0 ) in the number of emergency admissions for asthma.

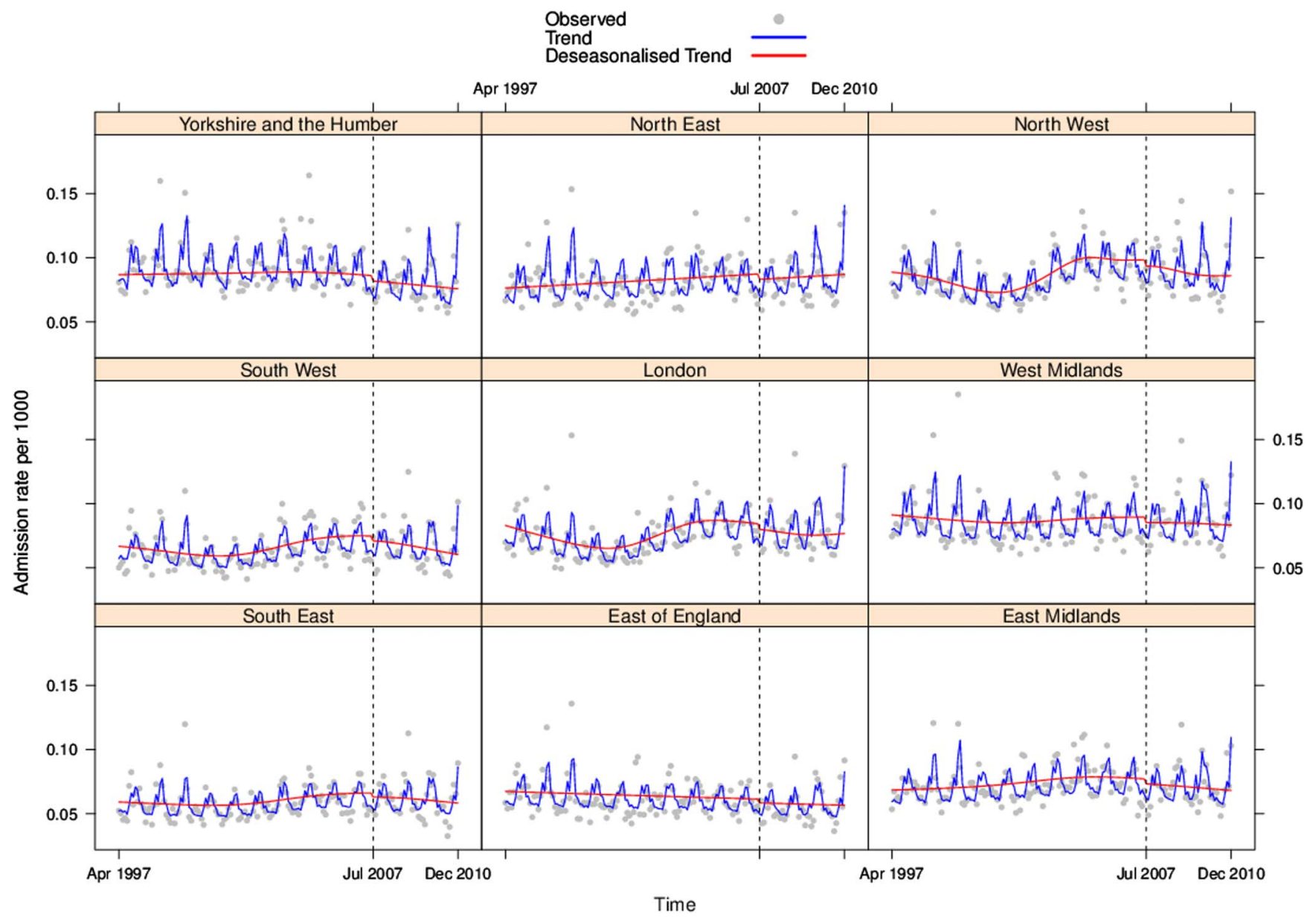

Figure 1 Monthly emergency hospital admission rate for asthma among adults. Observed (grey circles) monthly emergency hospital admission rate per 1000 adults for asthma in England by government office region during the period April 1997 to December 2010. The blue and red lines show the model estimated trend and de-seasonalised trend respectively (ie, with and without the seasonal components). The dashed vertical line indicates the date when smokefree legislation was introduced in England. This figure is only reproduced in colour in the online version. 
Table 1 Results of regression analysis to detect an association between smokefree legislation in England and emergency hospital admissions for asthma

\begin{tabular}{|c|c|c|c|c|}
\hline Effects* & edft & Parameter estimate & Standard error & $p$ value \\
\hline \multicolumn{5}{|l|}{ Parametric terms } \\
\hline \multicolumn{5}{|l|}{ Smokefree } \\
\hline \multicolumn{5}{|l|}{ Before§ } \\
\hline After & & -0.050 & 0.022 & 0.03 \\
\hline \multicolumn{5}{|l|}{ Region } \\
\hline \multicolumn{5}{|l|}{ North East§ } \\
\hline North West & & 0.050 & 0.022 & 0.02 \\
\hline Yorkshire and Humber & & 0.035 & 0.023 & 0.13 \\
\hline East Midlands & & -0.12 & 0.024 & $<0.001$ \\
\hline West Midlands & & 0.051 & 0.023 & 0.02 \\
\hline East of England & & -0.28 & 0.024 & $<0.001$ \\
\hline London & & -0.079 & 0.023 & $<0.001$ \\
\hline South East & & -0.31 & 0.023 & $<0.001$ \\
\hline South West & & -0.22 & 0.024 & $<0.001$ \\
\hline \multicolumn{5}{|l|}{ Smooth terms } \\
\hline Influenza & 3.58 & & $<0.001$ & \\
\hline Temperature & 5.53 & & 0.006 & \\
\hline Month & 7.71 & & $<0.001$ & \\
\hline \multicolumn{5}{|l|}{ Region×year } \\
\hline North East & 1.00 & & 0.008 & \\
\hline North West & 6.17 & & $<0.001$ & \\
\hline Yorkshire and Humber & 2.28 & & 0.21 & \\
\hline East Midlands & 2.79 & & 0.06 & \\
\hline West Midlands & 2.82 & & 0.32 & \\
\hline East of England & 1.00 & & 0.03 & \\
\hline London & 5.56 & & $<0.001$ & \\
\hline South East & 3.67 & & $<0.001$ & \\
\hline South West & 4.30 & & $<0.001$ & \\
\hline
\end{tabular}

Across England as a whole, we estimate that approximately 1900 emergency admissions for asthma were prevented in the first year post legislation, with a similar number of admissions prevented in the second and third years post legislation. The drop in admissions did not vary significantly across regions ( $p$ values for the interaction between the smokefree predictor and each region range from 0.29 to 0.85 ) and the interaction term between the smokefree legislation predictor and region was therefore not included in the final model. The residuals from our model exhibited no autocorrelation or partial autocorrelation and did not deviate significantly from model assumptions.

\section{Sensitivity analyses}

The first sensitivity analysis indicated that none of the false date predictors were significant $(p<0.05)$. The second sensitivity analysis indicated that our model is robust to the choice of length of the pre-legislation and post-legislation period in the range allowed by the model fitting (see online supplementary tables S1 and S2 and online supplementary appendix). The range specified by the CIs was very similar when we varied the length of the pre-legislation or post-legislation period, and the association was negative and statistically significant for all but one finding (using 6 years of pre-legislative data).

\section{DISCUSSION}

England's smoke-free legislation was associated with a statistically significant fall in emergency hospital admissions for asthma among adults. After adjusting for long-term trends in asthma and seasonality, the introduction of the ban was associated with a $4.9 \%$ reduction in admissions and this observed reduction was similar across geographical regions. This implies that almost 1900 emergency admissions for asthma were prevented in England during the first year after legislation was introduced and a similar number of emergency admissions for asthma were prevented in the subsequent 2 years. The decrease in emergency admissions for asthma among adults was lower than that observed in other countries. ${ }^{17-19} 22$ This may be attributable in part to the differences in the specification of the longterm trend between these studies and ours. Apart from attempting to account for underlying secular trends we also performed additional sensitivity analyses to provide further support for our findings. Differences could also be due, in part, to a smaller reduction in SHS exposure immediately following smokefree legislation in England than in other jurisdictions attributable to the fact that many workplaces were already smoke free. This is supported by data from New York, ${ }^{11}$ Scotland $^{12}$ and England, ${ }^{13}$ which showed 
geometric mean saliva cotinine levels in non-smoking adults declining by $47 \%, 39 \%$ and $27 \%$ respectively.

We identified a smaller reduction than that observed for children aged under 15 years old in England (9\%, 95\% CI 7\% to $11 \%),{ }^{28}$ although we are unable to conclude that there is a significant difference between the two estimates because there is some overlap in the $95 \%$ CIs. Our results are consistent with published work that the introduction of smokefree legislation in England is associated with a reduction in SHS exposure among non-smoking adults. ${ }^{13}$ In contrast, recent evidence suggests no corresponding reduction in SHS exposure among children. ${ }^{26}$ Assuming that the modelling assumptions in all the studies are correct, one possible explanation for the discrepancy is that there actually was an immediate reduction in SHS exposure among children after legislation was introduced but it was too small to be detected using available SHS exposure data. As children are still developing and have higher breathing rates than adults, they are more vulnerable to the effects of SHS and may show significant health benefits, even with a small change in SHS exposure. Furthermore, the majority of children are nonsmokers whereas in adults $20 \%$ are smokers and therefore a small change in SHS exposure has the potential to benefit a large proportion of children.

\section{Strengths and limitations}

This study has two major strengths. First, it is the largest study to date that has evaluated the impact of smokefree legislation on admissions for asthma in adults, with data collected on all admissions for the 43 million individuals aged 16 years or older who live in England, compared with less than 7 million in each of the other studies. This offers greater statistical power to detect smaller declines in admissions in a short time scale, as observed in England. Second, we used statistical methodology that appropriately adjusted for non-linear trends in admissions. As shown in a previous smokefree evaluation, incorrect assumptions concerning the shape of the trend could bias results considerably. ${ }^{23}$

Our results were also robust to the false data analysis and moderate changes in the length of the pre-legislative and postlegislative period. We did observe a non-significant effect of smokefree legislation when using only 6 years of pre-legislative data; this coincides with a noticeable change in the long-term trend for several regions (figure 1), which introduces additional variability causing the non-significant result.

There are a number of limitations. Although our study shows a statistically significant association between the introduction of smokefree legislation and emergency admissions for asthma in adults, it does not prove a causal relationship. While there are a number of other pieces of evidence that support a causal association $^{33}$ (eg, biological plausibility given the documented links between SHS exposure and airways responsiveness and exacerbations of asthma ${ }^{3-9} 34$ and biologically documented reductions in an adult's SHS exposure after legislation was introduced ${ }^{13}$ ), studies from other jurisdictions need to be published to validate our findings.

While we accounted for seasonality, variation in population size and underlying trends in admissions, there may also be unknown confounders that are related to the outcome (admissions for asthma) and that changed at the time of the legislation, which could have contributed to the observed decline. However, we cannot suggest other factors that have this relationship and have not been included in the model. In particular, we are not aware of changes in recommendations for the treatment of asthma or of any new drugs for asthma at that time.
In common with many studies from other jurisdictions that also investigated the impact of smokefree legislation on population health, we relied on routine hospital data, and while this meant we could adjust for long-term trends, it precluded our ability to analyse admissions among non-smokers only. How much of the reduction in admissions at the time that legislation was introduced, after adjusting for pre-legislative trends, was attributable to reduced SHS exposure and how much to reductions in active smoking is therefore not clear. Recent evidence showing that smokefree legislation in England is associated with a reduction in SHS exposure among non-smoking adults, ${ }^{13}$ but not smoking prevalence or consumption among smokers, ${ }^{35}$ suggests that most of the observed reduction in admissions is in fact attributable to reduced SHS exposure. Pell et $a l^{36}$ were able to examine the impacts of Scotland's smokefree legislation on hospital admissions for acute coronary events according to smoking status because they conducted a prospective study, rather than a retrospective study relying on routine hospital data. However, because this study adopted a before-after study design it could not take into account the potential effect of the long-term trend in acute coronary syndrome. Not taking into account long-term trends, particularly if those of smokers and non-smokers are different, could lead to biased estimates of the impact of legislation, and highlights the need for both approaches.

A further potential limitation concerns how admissions were assigned to regions, that is, based on the patient's residence, the concern being that the region of residence may in some instances differ from where the admitted patient was exposed to the 'cause'. This might apply for working populations, for example. However, the location of the hospital, which perhaps more closely matches with place of exposure, is often not recorded in HES, and given that this study evaluates the impact in large regions of the country, any disparity between region of patient's residence and region of patient's exposure is likely to affect only a small proportion of the population.

\section{CONCLUSIONS}

This study provides the first assessment of the impact of the legislation on hospital admissions for asthma among adults in England. It also makes an important contribution to the international debate on this topic by making a more thorough attempt to account for underlying secular trends than previous studies on this topic; and being the largest study to date, thereby overcoming the limitations of previous studies. In doing so, it provides further support to a growing body of national and international evidence of the positive effects that introducing smokefree policies has on public health.

Acknowledgements The HES data were made available by the NHS Health and Social Care Information Centre. We would like to thank the Public Health Observatory Network for providing access to data, in particular Bobbie Jacobson Julia Verne and Paul Brown. We are also grateful to Richard Hubbard for his comments on an earlier draft of this paper and to Ruth Salway for advice on the statistical analysis.

Contributors AG conceptualised the study, drafted and edited the paper; MS planned and undertook the analysis, drafted and edited the paper; RM helped prepare the data and edited the paper. All authors have approved the final version.

Funding This work was undertaken by the University of Bath which received funding from the Department of Health's Policy Research Programme. The views expressed in the publication are those of the authors and not necessarily those of the Department of Health. AG is supported by a Health Foundation Clinician Scientist Fellowship. MS and AG are members of the UK Centre for Tobacco Control Studies which receives core funding from the British Heart Foundation, Cancer Research UK, Economic and Social Research Council, Medical Research Council, and the National Institute of Health Research under the auspices of the UK Clinical Research Collaboration. 
The funders had no role in the study design, data collection and analysis, interpretation of data, decision to publish or preparation of the manuscript

Competing interests None.

Provenance and peer review Not commissioned; externally peer reviewed.

\section{REFERENCES}

1 Flouris $\mathrm{AD}$, Vardavas $\mathrm{Cl}$, Metsios $\mathrm{GS}$, et al. Biological evidence for the acute health effects of secondhand smoke exposure. Am J Physiol Lung Cell Mol Physiol 2010;298:L3-12.

2 Royal College of Physicians. Going smoke-free: the medical case for clean air in the home, at work and in public places. A report by the Tobacco Advisory Group of the Royal College of Physicians. London: RCP, 2005:35-7.

3 Knight A, Breslin AB. Passive cigarette smoking and patients with asthma. Med J Aust 1985;142:194-5.

4 US Department of Health and Human Services. The health consequences of involuntary exposure to tobacco smoke: a report of the surgeon general. Washington, DC: Department of Health and Human Services, 2006.

5 Britton J. Passive smoking and asthma exacerbation. Thorax 2005;60:794-5.

6 Eisner M, Balmes J, Yelin E, et al. Directly measured secondhand smoke exposure and COPD health outcomes. BMC Pulm Med 2006;6:12.

7 Stankus RP, Menon PK, Rando RJ, et al. Cigarette smoke-sensitive asthma: challenge studies. J Allerg Clin Immun 1988;82:331-8.

8 Nowak D, Jörres R, Schmidt $A$, et al. Effect of 3 hours' passive smoke exposure in the evening on airway tone and responsiveness until next morning. Int Arch Occup Environ Health 1996;69:125-33.

9 Menon P, Rando RJ, Stankus RP, et al. Passive cigarette smoke — challenge studies: increase in bronchial hyperreactivity. J Allerg Clin Immun 1992;89:560-6.

10 IARC. Chapter 6: Reductions in exposure to secondhand smoke and effects on health due to restrictions on smoking. IARC handbook of cancer prevention, tobacco control: evaluating the effectiveness of smoke-free policies. Lyon, France: WHO, IARC, 2009:135-62.

11 Bauer U, Juster $\mathrm{H}$, Hyland $\mathrm{A}$, et al. Reduced secondhand smoke exposure after implementation of a comprehensive statewide smoking ban New York, June 26, 2003 June 30, 2004. JAMA 2007:298:1392-4.

12 Haw SJ, Gruer L. Changes in exposure of adult non-smokers to secondhand smoke after implementation of smoke-free legislation in Scotland: national cross sectional survey. BMJ 2007;335:549-52

13 Sims M, Mindell JS, Jarvis MJ, et al. Did smokefree legislation in England reduce exposure to secondhand smoke among nonsmoking adults? Cotinine analysis from the health survey for England. Environ Health Perspect 2012;120:425-30.

14 Tan CE, Glantz SA. Association between smoke-free legislation and hospitalizations for cardiac, cerebrovascular, and respiratory diseases: a meta-analysis. Circulation 2012:126:2177-83

15 Dove MS, Dockery DW, Connolly GN. Smoke-free air laws and asthma prevalence, symptoms, and severity among nonsmoking youth. Pediatrics 2011;127:102-9.

16 Mackay D, Haw S, Ayres JG, et al. Smoke-free legislation and hospitalizations for childhood asthma. N Engl J Med 2010;363:1139-45.

17 Rayens MK, Burkhart PV, Zhang M, et al. Reduction in asthma-related emergency department visits after implementation of a smoke-free law. J Allerg Clin Immun 2008:122:537-41.e3.
18 Edwards E, Bullen C, O'Dea D, et al. After the smoke has cleared: evaluation of the impact of a new smokefree law. Wellington: New Zealand Ministry of Health, 2006.

19 Kent BD, Sulaiman I, Nicholson TT, et al. Acute pulmonary admissions following implementation of a national workplace smoking ban. Chest 2012;

142:673-9.

20 Herman PM, Walsh ME. Hospital admissions for acute myocardial infarction, angina, stroke, and asthma after implementation of Arizona's comprehensive statewide smoking ban. Am J Public Health 2011;101:491-6.

21 Naiman A, Glazier RH, Moineddin R. Association of anti-smoking legislation with rates of hospital admission for cardiovascular and respiratory conditions. CMAJ 2010;182:761-7.

22 Moraros J, Bird Y, Chen S, et al. The impact of the 2002 Delaware smoking ordinance on heart attack and asthma. Int I Environ Res Public Health 2010;7:4169-78.

23 Gasparrini A, Gorini G, Barchielli A. On the relationship between smoking bans and incidence of acute myocardial infarction. Eur I Epidemiol 2009; 24:597-602.

24 Department of Health. An outcomes strategy for people with chronic obstructive pulmonary disease (COPD) and asthma in England. London: Department of Health, 2011.

25 Asthma UK. Wish you were here? London: Asthma UK, 2008

26 Jarvis MJ, Sims M, Gilmore A, et al. Impact of smoke-free legislation on children's exposure to secondhand smoke: cotinine data from the Health Survey for England. Tob Control 2012;21:18-23.

27 Sims M, Bauld L, Gilmore A. England's legislation on smoking in indoor public places and workplaces: impact on the most exposed children. Addiction 2012;107:2009-16.

28 Millett C, Lee JT, Laverty AA, et al. Hospital admissions for childhood asthma after smoke-free legislation in England. Pediatrics 2013. Published Online First: 21 January 2013 doi: 10.1542/peds.2012-2592

29 NHS Information Centre for Health and Social Care. Hospital episode statistics. 2012. http://www.hesonline.nhs.uk/Ease/servlet/ContentServer?sitelD=1937 (accessed 13 Mar 2013).

30 World Health Organisation. International statistical classification of diseases and related health problems, Tenth Revision. Geneva: World Health Organisation, 1992.

31 Sims M, Maxwell R, Bauld L, et al. Short term impact of smoke-free legislation in England: retrospective analysis of hospital admissions for myocardial infarction. BMJ 2010;340:c2161.

32 Wood SN. Generalized additive models: an introduction with R. Boca Raton, FL: Chapman and Hall/CRC, 2006

33 Hill $A B$. The environment and disease: association or causation. Proc $R$ Soc Med 1965;58:295-300.

34 Flouris $\mathrm{AD}$, Vardavas $\mathrm{Cl}$, Metsios $\mathrm{GS}$, et al. Biological evidence for the acute health effects of secondhand smoke exposure. Am I Physiol Lung Cell Mol Physiol 2010;298:L3-12.

35 Lee JT, Glantz SA, Millett C. Effect of smoke-free legislation on adult smoking behaviour in England in the 18 months following implementation. PLoS One 2011;6:e20933.

36 Pell JP, Haw S, Cobbe S, et al. Smoke-free legislation and hospitalizations for acute coronary syndrome. N Engl J Med 2008;359:482-91. 